\title{
An exact representation of the fermion dynamics in terms of Poisson processes and its connection with Monte Carlo algorithms
}

\author{
Matteo Beccaria ${ }^{a}$ Carlo Presilla, ${ }^{b}$ Gian Fabrizio De Angelis, ${ }^{a}$ and Giovanni Jona-Lasinio ${ }^{b}$ \\ ${ }^{a}$ Dipartimento di Fisica, Università di Lecce, Via Arnesano, 73100 Lecce, Italy \\ ${ }^{b}$ Dipartimento di Fisica, Università di Roma "La Sapienza," Piazzale A. Moro 2, 00185 Roma, Italy \\ and INFM Unità di Ricerca di Roma "La Sapienza" \\ (submitted to Physical Review Letters, October 26, 1998)
}

\begin{abstract}
We present a simple derivation of a Feynman-Kac type formula to study fermionic systems. In this approach the real time or the imaginary time dynamics is expressed in terms of the evolution of a collection of Poisson processes. A computer implementation of this formula leads to a family of algorithms parametrized by the values of the jump rates of the Poisson processes. From these an optimal algorithm can be chosen which coincides with the Green Function Monte Carlo method in the limit when the latter becomes exact.
\end{abstract}

A crucial issue in quantum Monte Carlo methods is the choice of the most convenient stochastic process to be used in the simulation of the dynamics of the system. This aspect is particularly evident in the case of fermion systems [1] due to the anticommutativity of the variables involved which for long time have not lent themselves to direct numerical evaluation. In a recent paper [5] progress has been made in this direction by providing exact probabilistic expressions for quantities involving variables belonging to Grassmann or Clifford algebras. In particular, the real time or the imaginary time evolution operator of a Fermi system or a Berezin integral can be expressed in terms of an associated stochastic dynamics of a collection of Poisson processes. This approach depends on an older general formula to represent probabilistically the solution of a system of ordinary differential equations (ODE) in terms of Poisson processes [8]. In this paper, we present a simple derivation of a similar formula to study fermionic systems, in particular, the Hubbard model. However, the fermionic nature of the Hamiltonian plays no special role and similar representations can be written down for any system described by a finite Hamiltonian matrix. A computer implementation of this formula leads to a family of algorithms parametrized by the values of the jump rates of the Poisson processes. For an optimal choice of these parameters we obtain an algorithm which coincides with the well known Green Function Monte Carlo method in the limit when the latter becomes exact [6]. In this way we provide a theoretical characterization of GFMC.

Let us consider the Hubbard Hamiltonian

$$
\begin{aligned}
H= & -\sum_{i=1}^{|\Lambda|} \sum_{j=i+1}^{|\Lambda|} \sum_{\sigma=\uparrow \downarrow} \eta_{i j}\left(c_{i \sigma}^{\dagger} c_{j \sigma}+c_{j \sigma}^{\dagger} c_{i \sigma}\right) \\
& +\sum_{i=1}^{|\Lambda|} \gamma_{i} c_{i \uparrow}^{\dagger} c_{i \uparrow} c_{i \downarrow}^{\dagger} c_{i \downarrow},
\end{aligned}
$$

where $\Lambda \subset Z^{d}$ is a finite $d$-dimensional lattice with cardi- nality $|\Lambda|,\{1, \ldots,|\Lambda|\}$ some total ordering of the lattice points, and $c_{i \sigma}$ the usual anticommuting destruction operators at site $i$ and spin index $\sigma$. In this paper, we are interested in evaluating the matrix elements $\left\langle\boldsymbol{n}^{\prime}\left|e^{-H t}\right| \boldsymbol{n}\right\rangle$ where $\boldsymbol{n}=\left(n_{1 \uparrow}, n_{1 \downarrow}, \ldots, n_{|\Lambda| \uparrow}, n_{|\Lambda| \downarrow}\right)$ are the occupation numbers taking the values 0 or 1 [7]. The total number of fermions per spin component is a conserved quantity, therefore we consider only configurations $\boldsymbol{n}$ and $\boldsymbol{n}^{\prime}$ such that $\sum_{i=1}^{|\Lambda|} n_{i \sigma}^{\prime}=\sum_{i=1}^{|\Lambda|} n_{i \sigma}$ for $\sigma=\uparrow \downarrow$. In the following we shall use the mod 2 addition $n \oplus n^{\prime}=\left(n+n^{\prime}\right) \bmod 2$.

Let $\Gamma=\left\{(i, j), 1 \leq i<j \leq|\Lambda|: \eta_{i j} \neq 0\right\}$ and $|\Gamma|$ its cardinality. For simplicity, we will assume that $\eta_{i j}=\eta$ if $(i, j) \in \Gamma$ and $\gamma_{i}=\gamma$. By introducing

$$
\begin{aligned}
\lambda_{i j \sigma}(\boldsymbol{n}) \equiv & \left\langle\boldsymbol{n} \oplus \mathbf{1}_{i \sigma} \oplus \mathbf{1}_{j \sigma}\left|c_{i \sigma}^{\dagger} c_{j \sigma}+c_{j \sigma}^{\dagger} c_{i \sigma}\right| \boldsymbol{n}\right\rangle \\
= & (-1)^{n_{i \sigma}+\cdots+n_{j-1 \sigma}} \\
& \times\left[n_{j \sigma}\left(n_{i \sigma} \oplus 1\right)-n_{i \sigma}\left(n_{j \sigma} \oplus 1\right)\right],
\end{aligned}
$$

where $\mathbf{1}_{i \sigma}=\left(0, \ldots, 0,1_{i \sigma}, 0, \ldots, 0\right)$, and

$$
V(\boldsymbol{n}) \equiv\langle\boldsymbol{n}|H| \boldsymbol{n}\rangle=\gamma \sum_{i=1}^{|\Lambda|} n_{i \uparrow} n_{i \downarrow},
$$

the following representation holds

$$
\begin{gathered}
\left\langle\boldsymbol{n}^{\prime}\left|e^{-H t}\right| \boldsymbol{n}\right\rangle=\boldsymbol{E}\left(\delta_{\boldsymbol{n}^{\prime}, \boldsymbol{n} \oplus \boldsymbol{N}^{t}} \mathcal{M}^{t}\right) \\
\mathcal{M}^{t}=\exp \left\{\sum_{(i, j) \in \Gamma} \sum_{\sigma=\uparrow \downarrow} \int_{[0, t)} \log \left[\eta \rho^{-1} \lambda_{i j \sigma}\left(\boldsymbol{n} \oplus \boldsymbol{N}^{s}\right)\right] d N_{i j \sigma}^{s}\right. \\
\left.-\int_{0}^{t} V\left(\boldsymbol{n} \oplus \boldsymbol{N}^{s}\right) d s+2|\Gamma| \rho t\right\} .
\end{gathered}
$$

Here, $\left\{N_{i j \sigma}^{t}\right\},(i, j) \in \Gamma$, is a family of $2|\Gamma|$ independent Poisson processes with parameter $\rho$ and $\boldsymbol{N}^{t}=$ $\left(N_{1 \uparrow}^{t}, N_{1 \downarrow}^{t}, \ldots, N_{|\Lambda| \uparrow}^{t}, N_{|\Lambda| \downarrow}^{t}\right)$ are $2|\Lambda|$ stochastic processes defined as 


$$
N_{k \sigma}^{t}=\sum_{(i, j) \in \Gamma:} N_{i=k \text { or } j=k}^{t} .
$$

We remind that a Poisson process $N^{t}$ with parameter $\rho$ is a jump process characterized by the following probabilities:

$$
P\left(N^{t+s}-N^{t}=k\right)=\frac{(\rho s)^{k}}{k !} e^{-\rho s} .
$$

Its trajectories are piecewise-constant increasing integervalued functions continuous from the left. The stochastic integral $\int d N^{t}$ is just an ordinary Stieltjes integral

$$
\int_{[0, t)} f\left(s, N^{s}\right) d N^{s}=\sum_{k: s_{k}<t} f\left(s_{k}, N^{s_{k}}\right),
$$

where $s_{k}$ are random jump times having probability density $p(s)=\rho e^{-\rho s}$. Finally, the symbol $\boldsymbol{E}(\ldots)$ is the expectation of the stochastic functional within braces. We emphasize that a similar representation holds for the real time matrix elements $\left\langle\boldsymbol{n}^{\prime}\left|e^{-i H t}\right| \boldsymbol{n}\right\rangle$.

Summarizing, we associate to each $\eta_{i j} \neq 0$ a link connecting the sites $i$ and $j$ and assign to it a pair of Poisson processes $N_{i j \sigma}^{t}$ with $\sigma=\uparrow \downarrow$. Then, we assign to each site $i$ and spin component $\sigma$ a stochastic process $N_{i \sigma}^{t}$ which is the sum of all the processes associated with the links incoming at that site and having the same spin component. A jump in the link process $N_{i j \sigma}^{t}$ implies a jump in both the site processes $N_{i \sigma}^{t}$ and $N_{j \sigma}^{t}$. Equations (4) and (5) are immediately generalizable to non identical parameters $\eta_{i j}$ and $\gamma_{i}$. In this case, it may be convenient to use Poisson processes $N_{i j \sigma}^{t}$ with different parameters $\rho_{i j \sigma}$.

We now show that the representation (45) follows from the general formula to represent probabilistically the solution of an ODE system [8] and the expression of the matrix elements of $H$. The matrix elements $\left\langle\boldsymbol{n}^{\prime}\left|e^{-H t}\right| \boldsymbol{n}\right\rangle$ obey the ODE system

$$
\frac{d}{d t}\left\langle\boldsymbol{n}^{\prime}\left|e^{-H t}\right| \boldsymbol{n}\right\rangle=-\sum_{\boldsymbol{n}^{\prime \prime}}\left\langle\boldsymbol{n}^{\prime}|H| \boldsymbol{n}^{\prime \prime}\right\rangle\left\langle\boldsymbol{n}^{\prime \prime}\left|e^{-H t}\right| \boldsymbol{n}\right\rangle .
$$

One may check that (田司) is indeed solution of (8) by applying the rules of stochastic differentiation. We have

$$
\begin{aligned}
\boldsymbol{E} & \left(\delta_{\boldsymbol{n}^{\prime}, \boldsymbol{n} \oplus \boldsymbol{N}^{t+d t}} \mathcal{M}^{t+d t}\right) \\
= & \sum_{\boldsymbol{n}^{\prime \prime}} \boldsymbol{E}\left(\prod_{(i, j) \in \Gamma} \prod_{\sigma=\uparrow \downarrow} \delta_{\boldsymbol{n}^{\prime}, \boldsymbol{n}^{\prime \prime} \oplus d \boldsymbol{N}^{t}}\right. \\
& \times e^{\int_{[t, t+d t)} \log \left[\eta \rho^{-1} \lambda_{i j \sigma}\left(\boldsymbol{n} \oplus \boldsymbol{N}^{s}\right)\right] d N_{i j \sigma}^{s}} \\
& \left.\times e^{-V\left(\boldsymbol{n} \oplus \boldsymbol{N}^{t}\right) d t+2|\Gamma| \rho d t} \delta_{\boldsymbol{n}^{\prime \prime}, \boldsymbol{n} \oplus \boldsymbol{N}^{t} \mathcal{M}^{t}}\right)
\end{aligned}
$$

For the Markov property, the expectation of the factors containing the stochastic integrals in the interval $[t, t+d t]$ can be taken separately. By expanding each one of them over all possible numbers of jumps of the Poisson processes as

$$
\begin{aligned}
& \boldsymbol{E}\left(\delta_{\boldsymbol{n}^{\prime}, \boldsymbol{n}^{\prime \prime} \oplus d \boldsymbol{N}^{t}} e^{\int_{[t, t+d t)} \log \left[\eta \rho^{-1} \lambda_{i j \sigma}\left(\boldsymbol{n} \oplus \boldsymbol{N}^{s}\right)\right] d N_{i j \sigma}^{s}}\right) \\
& =\delta_{\boldsymbol{n}^{\prime}, \boldsymbol{n}^{\prime \prime}} e^{0} e^{-\rho d t} \\
& +\delta_{\boldsymbol{n}^{\prime}, \boldsymbol{n}^{\prime \prime} \oplus \mathbf{1}_{i \sigma} \oplus \mathbf{1}_{j \sigma}} e^{\log \left[\eta \rho^{-1} \lambda_{i j \sigma}\left(\boldsymbol{n} \oplus \boldsymbol{N}^{t}\right)\right]} e^{-\rho d t} \rho d t+\ldots \\
& =\delta_{\boldsymbol{n}^{\prime}, \boldsymbol{n}^{\prime \prime}}+\left[\delta_{\boldsymbol{n}^{\prime}, \boldsymbol{n}^{\prime \prime} \oplus \mathbf{1}_{i \sigma} \oplus \mathbf{1}_{j \sigma}} \eta \rho^{-1} \lambda_{i j \sigma}\left(\boldsymbol{n} \oplus \boldsymbol{N}^{t}\right)\right. \\
& \left.-\delta_{\boldsymbol{n}^{\prime}, \boldsymbol{n}^{\prime \prime}}\right] \rho d t+\mathcal{O}\left(d t^{2}\right),
\end{aligned}
$$

up to order $d t$ we obtain

$$
\begin{aligned}
\boldsymbol{E} & \left(\delta_{\boldsymbol{n}^{\prime}, \boldsymbol{n} \oplus \boldsymbol{N}^{t+d t}} \mathcal{M}^{t+d t}\right) \\
= & \sum_{\boldsymbol{n}^{\prime \prime}}\left[\delta_{\boldsymbol{n}^{\prime}, \boldsymbol{n}^{\prime \prime}}+\sum_{(i, j) \in \Gamma} \sum_{\sigma=\uparrow \downarrow} \delta_{\boldsymbol{n}^{\prime}, \boldsymbol{n}^{\prime \prime} \oplus \mathbf{1}_{i \sigma} \oplus \mathbf{1}_{j \sigma}} \eta \lambda_{i j \sigma}\left(\boldsymbol{n}^{\prime \prime}\right) d t\right. \\
& \left.-\delta_{\boldsymbol{n}^{\prime}, \boldsymbol{n}^{\prime \prime}} V\left(\boldsymbol{n}^{\prime \prime}\right) d t\right] \boldsymbol{E}\left(\delta_{\boldsymbol{n}^{\prime \prime}, \boldsymbol{n} \oplus \boldsymbol{N}^{t}} \mathcal{M}^{t}\right) .
\end{aligned}
$$

Finally, we rewrite this relation as

$$
\begin{aligned}
d \boldsymbol{E} & \left(\delta_{\boldsymbol{n}^{\prime}, \boldsymbol{n} \oplus \boldsymbol{N}^{t}} \mathcal{M}^{t}\right) \\
& =\boldsymbol{E}\left(\delta_{\boldsymbol{n}^{\prime}, \boldsymbol{n} \oplus \boldsymbol{N}^{t+d t}} \mathcal{M}^{t+d t}\right)-\boldsymbol{E}\left(\delta_{\boldsymbol{n}^{\prime}, \boldsymbol{n} \oplus \boldsymbol{N}^{t}} \mathcal{M}^{t}\right) \\
& =-\sum_{\boldsymbol{n}^{\prime \prime}}\left\langle\boldsymbol{n}^{\prime}|H| \boldsymbol{n}^{\prime \prime}\right\rangle \boldsymbol{E}\left(\delta_{\boldsymbol{n}^{\prime \prime}, \boldsymbol{n} \oplus \boldsymbol{N}^{t}} \mathcal{M}^{t}\right) d t .
\end{aligned}
$$

It is clear that the fermionic nature of the Hamiltonian $H$ plays no special role in the above derivation. For later use, note that summing (11) over $\boldsymbol{n}^{\prime}$ we have $d \boldsymbol{E}\left(\mathcal{M}^{t}\right)=-\boldsymbol{E}\left(\mathcal{H}^{t} \mathcal{M}^{t}\right) d t$, where

$$
\begin{aligned}
\mathcal{H}^{t} & \equiv \sum_{\boldsymbol{n}^{\prime}}\left\langle\boldsymbol{n}^{\prime}|H| \boldsymbol{n} \oplus \boldsymbol{N}^{t}\right\rangle \\
& =-\eta \sum_{(i, j) \in \Gamma} \sum_{\sigma=\uparrow \downarrow} \lambda_{i j \sigma}\left(\boldsymbol{n} \oplus \boldsymbol{N}^{t}\right)+V\left(\boldsymbol{n} \oplus \boldsymbol{N}^{t}\right) .
\end{aligned}
$$

In order to construct an efficient algorithm for evaluating (4.5), we start by observing that the functions $\lambda_{i j \sigma}\left(\boldsymbol{n} \oplus \boldsymbol{N}^{s}\right)$ vanish when the occupation numbers $n_{i \sigma} \oplus N_{i \sigma}^{s}$ and $n_{j \sigma} \oplus N_{j \sigma}^{s}$ are equal. We say that for a given value of $\sigma$ the link $i j$ is active at time $s$ if $\lambda_{i j \sigma}\left(\boldsymbol{n} \oplus \boldsymbol{N}^{s}\right) \neq 0$. We shall see in a moment that only active links are relevant. Let us consider how the stochastic integral in (5) builds up along a trajectory defined by considering the time ordered succession of jumps in the family $\left\{N_{i j \sigma}^{t}\right\}$. The contribution to the stochastic integral in the exponent of (5) at the first jump time of a link, for definiteness suppose that the link $i_{1} j_{1}$ with spin component $\sigma_{1}$ jumps first at time $s_{1}$, is

$$
\log \left[\eta \rho^{-1} \lambda_{i_{1} j_{1} \sigma_{1}}\left(\boldsymbol{n} \oplus \boldsymbol{N}^{s_{1}}\right)\right] \theta\left(t-s_{1}\right),
$$

where $\boldsymbol{N}^{s_{1}}=\mathbf{0}$ due the assumed left continuity. Therefore, if the link $i_{1} j_{1} \sigma_{1}$ was active at time 0 we obtain a finite contribution to the stochastic integral otherwise we obtain $-\infty$. If $s_{1} \geq t$ we have no contribution to the 
stochastic integral from this trajectory. If $s_{1}<t$ a second jump of a link, suppose $i_{2} j_{2}$ with spin component $\sigma_{2}$, can take place at time $s_{2}>s_{1}$ and we obtain a contribution

$$
\log \left[\eta \rho^{-1} \lambda_{i_{2} j_{2} \sigma_{2}}\left(\boldsymbol{n} \oplus \boldsymbol{N}^{s_{2}}\right)\right] \theta\left(t-s_{2}\right) .
$$

The analysis can be repeated by considering an arbitrary number of jumps. Of course, when the stochastic integral is $-\infty$, which is the case when some $\lambda=0$, there is no contribution to the expectation. The other integral in (5) is an ordinary integral of a piecewise constant bounded function.

We now describe the algorithm. From the above remarks it is clear that the only trajectories to be considered are those associated to the jumps of active links. It can be seen that this corresponds to the conservation of the total number of fermions per spin component. The active links can be determined after each jump by inspecting the occupation numbers of the sites in the set $\Gamma$ according to the rule that the link $i j$ is active for the spin component $\sigma$ if $n_{i \sigma}+n_{j \sigma}=1$. We start by determining the active links in the initial configuration $\boldsymbol{n}$ assigned at time 0 and make an extraction with uniform distribution to decide which of them jumps first. We then extract the jump time $s_{1}$ according to the probability density $p_{A_{1}}(s)=A_{1} \rho \exp \left(-A_{1} \rho s\right)$ where $A_{1}$ is the number of active links before the first jump takes place. The contribution to $\mathcal{M}^{t}$ at the time of the first jump is therefore, up to the factor $\exp (-2|\Gamma| \rho t)$,

$$
\begin{aligned}
& \eta \rho^{-1} \lambda_{i_{1} j_{1} \sigma_{1}}\left(\boldsymbol{n} \oplus \boldsymbol{N}^{s_{1}}\right) e^{-V\left(\boldsymbol{n} \oplus \boldsymbol{N}^{s_{1}}\right) s_{1}} e^{-\left(2|\Gamma|-A_{1}\right) \rho s_{1}} \\
& \times \theta\left(t-s_{1}\right)+e^{-V\left(\boldsymbol{n} \oplus \boldsymbol{N}^{t}\right) t} e^{-\left(2|\Gamma|-A_{1}\right) \rho t} \theta\left(s_{1}-t\right),
\end{aligned}
$$

where $\exp \left[-\left(2|\Gamma|-A_{1}\right) \rho s\right]$ is the probability that the $2|\Gamma|-A_{1}$ non active links do not jump in the time interval $s$. The contribution of a given trajectory is obtained by multiplying the factors corresponding to the different jumps until the last jump takes place later than $t$. For a given trajectory we thus have

$$
\begin{aligned}
\mathcal{M}^{t}=\prod_{k \geq 1}[ & \eta \rho^{-1} \lambda_{i_{k} j_{k} \sigma_{k}}\left(\boldsymbol{n} \oplus \boldsymbol{N}^{s_{k}}\right) \\
& \times e^{\left[A_{k} \rho-V\left(\boldsymbol{n} \oplus \boldsymbol{N}^{s_{k}}\right)\right]\left(s_{k}-s_{k-1}\right)} \theta\left(t-s_{k}\right) \\
& \left.+e^{\left[A_{k} \rho-V\left(\boldsymbol{n} \oplus \boldsymbol{N}^{t}\right)\right]\left(t-s_{k-1}\right)} \theta\left(s_{k}-t\right)\right] .
\end{aligned}
$$

Here, $A_{k}=A\left(\boldsymbol{n} \oplus \boldsymbol{N}^{s_{k}}\right)$ is the number of active links in the interval $\left(s_{k-1}, s_{k}\right]$ and $s_{0}=0$. Note that the exponentially increasing factor $\exp (2|\Gamma| \rho t)$ in (5) cancels out in the final expression of $\mathcal{M}^{t}$. The analogous expression of $\mathcal{M}^{t}$ for real times is simply obtained by replacing $\eta \rightarrow i \eta$ and $\gamma \rightarrow i \gamma$.

Let us specialize the algorithm for the calculation of the ground state energy $E_{0}$. This can be related to the matrix elements (4) in the following way

$$
E_{0}=-\lim _{t \rightarrow \infty} \frac{\sum_{\boldsymbol{n}^{\prime}} \partial_{t}\left\langle\boldsymbol{n}^{\prime}\left|e^{-H t}\right| \boldsymbol{n}\right\rangle}{\sum_{\boldsymbol{n}^{\prime}}\left\langle\boldsymbol{n}^{\prime}\left|e^{-H t}\right| \boldsymbol{n}\right\rangle} .
$$

The denominator in this expression corresponds to evaluating the expectation in (4) without the $\delta$ and is estimated by

$$
\sum_{\boldsymbol{n}^{\prime}}\left\langle\boldsymbol{n}^{\prime}\left|e^{-H t}\right| \boldsymbol{n}\right\rangle \simeq \frac{1}{K} \sum_{p=1}^{K} \mathcal{M}_{p}^{t}
$$

where the index $p$ denotes one of the $K$ simulated trajectories and $\mathcal{M}_{p}^{t}$ is the value of $\mathcal{M}^{t}$ for the $p$ th trajectory. The numerator of (14) is estimated similarly to $(15)$ with $\mathcal{M}_{p}^{t}$ replaced by $\mathcal{H}_{p}^{t} \mathcal{M}_{p}^{t}$, where $\mathcal{H}_{p}^{t}$ is the value for the $p$ th trajectory of $\mathcal{H}^{t}$ given by (12).

The variance of the stochastic process $\mathcal{M}^{t}$ increases with $t$ and its distribution is not well estimated if the number $K$ of trajectories remains fixed. As an alternative to increasing $K$, one may resort to the reconfiguration method [9]. A simulation with $K$ trajectories is performed for a time $t$ but is repeated $R$ times choosing randomly the initial configurations among those reached in the previous simulation [4, 11.

In principle, the algorithms parametrized by $\rho$ are all equivalent as (45) holds for any choice of the Poisson rates. However, since we estimate the expectation values with a finite number of trajectories, this may introduce a systematic error. In Fig. 1 we show the dependence of the error $E_{0}-E_{0}^{\text {exact }}$ as a function of $\rho$ in the case of a small one-dimensional system which can be exactly diagonalized. It is evident that the best performance of the algorithm is in correspondence of the natural choice $\rho \sim \eta$ independently of the interaction strength. This behavior can be understood on the basis of the following qualitative argument. The average number of configuration changes in the time $t$ is $A \rho t$ where $A$ is the average number of active links. This is also the average number of terms in the product of $(13)$. In absence of sign problem as in the case of Fig. 1, we roughly estimate the r.h.s. of (15) as $\left(\eta \rho^{-1}\right)^{A \rho t} e^{(A \rho-V) t}$, where $V$ is the average potential. We see that the derivative with respect to $\rho$ of this expression vanishes for $\rho=\eta$. It is easily seen that this corresponds also to a minimum of the variance of $\mathcal{M}^{t}$ and, therefore, to a minimum of the statistical error.

The convergence features of the algorithm can be improved also using the importance sampling method [10]. Consider the operator $\widetilde{H}$ isospectral to $H$ defined by the matrix elements $\left\langle\boldsymbol{n}^{\prime}|\widetilde{H}| \boldsymbol{n}\right\rangle=\left\langle\boldsymbol{n}^{\prime} \mid g\right\rangle\left\langle\boldsymbol{n}^{\prime}|H| \boldsymbol{n}\right\rangle\langle\boldsymbol{n} \mid g\rangle^{-1}$, where $|g\rangle$ is a given state. The stochastic representation (45) and the corresponding algorithmic implementation hold unchanged for the new operator $\widetilde{H}$ with the substitution $\lambda_{i j \sigma}(\boldsymbol{n}) \rightarrow \lambda_{i j \sigma}(\boldsymbol{n})\left\langle\boldsymbol{n} \oplus \mathbf{1}_{i \sigma} \oplus \mathbf{1}_{j \sigma} \mid g\right\rangle\langle\boldsymbol{n} \mid g\rangle^{-1}$. In this way, however, the value of $\rho$ is not tuned to obtain the best performance. For this purpose we have to choose $\rho$ dependent on the indices $i j \sigma$ and on the configuration $\boldsymbol{n}$ so that $\rho_{i j \sigma}(\boldsymbol{n})=\eta\left|\left\langle\boldsymbol{n} \oplus \mathbf{1}_{i \sigma} \oplus \mathbf{1}_{j \sigma} \mid g\right\rangle\langle\boldsymbol{n} \mid g\rangle^{-1}\right|$. 
We consider now the connection of our approach with the GFMC method. In the GFMC, the ground state of the system is filtered out from a given initial state $|\boldsymbol{n}\rangle$ by iteratively applying the operator $G=1-H \Omega^{-1}$. In the occupation number representation we can write

$$
\left\langle\boldsymbol{n}^{\prime}|G| \boldsymbol{n}\right\rangle=\mathcal{P}_{\boldsymbol{n}^{\prime} \boldsymbol{n}} b_{\boldsymbol{n}^{\prime} \boldsymbol{n}},
$$

where

$$
\mathcal{P}_{\boldsymbol{n}^{\prime} \boldsymbol{n}}=\frac{\left|\left\langle\boldsymbol{n}^{\prime}|G| \boldsymbol{n}\right\rangle\right|}{\sum_{\boldsymbol{n}^{\prime \prime}}\left|\left\langle\boldsymbol{n}^{\prime \prime}|G| \boldsymbol{n}\right\rangle\right|}
$$

is a stochastic matrix and

$$
b_{\boldsymbol{n}^{\prime} \boldsymbol{n}}=\frac{\left\langle\boldsymbol{n}^{\prime}|G| \boldsymbol{n}\right\rangle}{\left|\left\langle\boldsymbol{n}^{\prime}|G| \boldsymbol{n}\right\rangle\right|} \sum_{\boldsymbol{n}^{\prime \prime}}\left|\left\langle\boldsymbol{n}^{\prime \prime}|G| \boldsymbol{n}\right\rangle\right|
$$

a, possibly negative, weight factor. A trajectory is defined as a series of steps, each one of duration $\Omega^{-1}$, in which the configuration changes from $\boldsymbol{n}$ to $\boldsymbol{n}^{\prime}$ according to the stochastic matrix $\mathcal{P}_{\boldsymbol{n}^{\prime} \boldsymbol{n}}$. The weight of a trajectory is $\prod_{\{\boldsymbol{n}\}} b_{\boldsymbol{n}^{\prime} \boldsymbol{n}}$, where $\boldsymbol{n}$ runs over the intermediate configurations visited by the trajectory [4]. Under our hypothesis $\eta_{i j}=\eta$ if $(i, j) \in \Gamma$, the GFMC algorithm becomes very simple because all the active links have the same probability to jump.

For large values of $\Omega$ the jump probability vanishes and the GFMC as described above becomes inefficient. As remarked in [1], one can cope with this difficulty in the following way. The probability that a configuration $\boldsymbol{n}$ changes at the step $n_{s}+1$, being unchanged in the previous $n_{s}$ steps, is $p\left(n_{s}\right)=\left(\mathcal{P}_{\boldsymbol{n n}}\right)^{n_{s}}\left(1-\mathcal{P}_{\boldsymbol{n} \boldsymbol{n}}\right)$. Therefore, we can directly assign the weight

$$
\left(b_{\boldsymbol{n} \boldsymbol{n}}\right)^{n_{s}} b_{\boldsymbol{n}^{\prime} \boldsymbol{n}}=\lambda_{i j \sigma}(\boldsymbol{n})\left\{1+[\eta A(\boldsymbol{n})-V(\boldsymbol{n})] \Omega^{-1}\right\}^{n_{s}+1}
$$

to a configuration change $\boldsymbol{n} \rightarrow \boldsymbol{n}^{\prime}=\boldsymbol{n} \oplus \mathbf{1}_{i \sigma} \oplus \mathbf{1}_{j \sigma}$ in which a fermion with spin $\sigma$ is moved from the site $i$ to the site $j$ or viceversa. As before, $A(\boldsymbol{n})$ is the number of active links in the configuration $\boldsymbol{n}$ and $\lambda_{i j \sigma}(\boldsymbol{n})$ and $V(\boldsymbol{n})$ are given by (2) and (3), respectively. The random integer $n_{s}$ is extracted with probability $p\left(n_{s}\right)$, e.g. $\quad n_{s}=\left\lfloor\log r / \log \mathcal{P}_{\boldsymbol{n n}}\right\rfloor$, where $r$ is a random number with uniform distribution in $[0,1]$. Note that the distribution of $n_{s}$ becomes Poissonian with parameter $-\log \mathcal{P}_{\boldsymbol{n} \boldsymbol{n}} \simeq \eta A(\boldsymbol{n}) \Omega^{-1}$ for $\Omega$ large. At this level, there is no apparent connection between this Poisson process and those entering in our formula (5). However, as suggested in [6] one can take the continuum limit $\Omega^{-1} \rightarrow 0$ and reconstruct in this way the semigroup $\exp (-H t)$. It is easy to verify that in this limit the GFMC algorithm coincides with that obtained from our formula for the optimal choice $\rho=\eta$. In fact, the time interval before a configuration change takes place is $s=n_{s} \Omega^{-1}$. For $\Omega^{-1} \rightarrow 0$, the random variable $s$ becomes continuously distributed in $[0, \infty)$ with probability density $p(s)=\Omega p\left(n_{s}\right)=\eta A(\boldsymbol{n}) \exp [-\eta A(\boldsymbol{n}) s]$ and the trajectory weight corresponding to the interval $s$ reduces to $\lambda_{i j \sigma}(\boldsymbol{n}) \exp \{[\eta A(\boldsymbol{n})-V(\boldsymbol{n})] s\}$. The coincidence holds also when importance sampling is included. If we change in Eqs. (16-18) $\Omega$ into $-i \Omega$ and interpret the absolute value as the modulus, in the continuum limit $\Omega^{-1} \rightarrow 0$ the extended GFMC algorithm coincides with our algorithm also for real times.

Partial support of INFN, Iniziativa Specifica RM42, is acknowledged.

[1] N. Trivedi and D. M. Ceperley, Phys. Rev. B 41, 4552 (1990).

[2] W. von der Linden, Phys. Rep. 220, 53 (1992).

[3] S. Zhang, J. Carlson, and J. E. Gubernatis, Phys. Rev. B 55, 7464 (1997).

[4] M. Calandra Buonaura and S. Sorella, Phys. Rev. B 57, 11446 (1998).

[5] G. F. De Angelis, G. Jona-Lasinio, and V. Sidoravicius, J. Phys. A 31, 289 (1998).

[6] M. Calandra, F. Becca, and S. Sorella, Phys. Rev. Lett. 81, 5185 (1998).

[7] We refer to $t$ as the physical time even if it is, properly speaking, an imaginary physical time.

[8] G. F. De Angelis, G. Jona-Lasinio, and M. Sirugue, J. Phys. A 16, 2433 (1983).

[9] J. H. Hetherington, Phys. Rev. A 30, 2713 (1984).

[10] D. M. Ceperley and M. H. Kalos, in Monte Carlo Methods in Statistical Physics, edited by K. Binder (SpringerVerlag, Heidelberg, 1992).

[11] S. Sorella, Phys. Rev. Lett. 80, 4558 (1998).

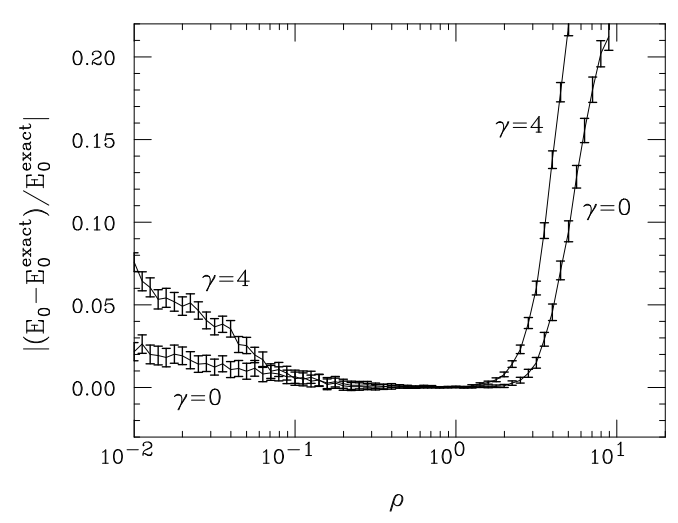

FIG. 1. Absolute value of the relative error $\left(E_{0}-E_{0}^{\text {exact }}\right) / E_{0}^{\text {exact }}$ on the ground state energy of an interacting $(\gamma=4)$ and noninteracting $(\gamma=0)$ Hubbard system obtained with the present algorithm as a function of the Poisson parameter $\rho$. The system is one-dimensional with periodic boundary conditions, $|\Lambda|=8, \sum_{i=1}^{|\Lambda|} n_{i \uparrow}=\sum_{i=1}^{|\Lambda|} n_{i \downarrow}=3$, $\eta_{i j}=1 \times \delta_{i j-1}$, and $\gamma_{i}=4$ or $\gamma_{i}=0$. In the simulation we used $K=10^{3}, R=10^{4}$ and $t=0.26$. Error bars represent statistical errors. 